\title{
Pacto Andino y Libre Comercio
}

A comienzos de septiembre, cuando se escriben estals lineas, subsiste un grave problema en el seno ciel Pacto Andino, que puede culminar con la salida de Chile del proceso que agrupa a seis países. Los otros cinco-Bolivia, Colombia, Ecuador, Perú y Venezuela- Ian logrado un acuerdo en el campo arancelatio y' en el tratamiento a la inversión extranjera. De esta manern, ias reiteradas informaciones difundidas con anterioridad, de que habría diferencias fuertes entre esos paises aparecen desmentidas por los hechos. En consecuencia, se estarian desvaneciendo las esperanzas del equipo económico gubemamental de ser acompañados por otros países en las posiciones de libre comercio y de apertura incliscriminala a ia inversión extranjera. De materializarse esas esperanzas, el desarrollo probable sería la detención o el fracaso del proceso de integración. La cvolución actual indica, en cambio, que el proceso concinuaría, con todos sus miembros o sólo con cinco tle ellos. En lo que sigue examinaremos sucintamente el origen principal del problema $y$ los peligros que un desenlace negativo representaria para los intereses permanentes de Chile.

EI, FONDO DER, PIOHIENA

El origen de las difercncias entre representantes de nuestro pais y Ios restantes yace en lat orientación teórica dominante sin contrapeso entre los primeros. Cualquicr conocedor de la iiteratura cconómica sabe que, para esa concepción teórical, el libre comercio constituye un ideal al cual debe traturse de llegar lo antes posible. Los sostenedores de esa concepción teórica posecn una extrema confianza en el buen funcionamiento del mercalo, achacíndole sus deficiencias a la intervención estatal, Por último, tienen una visión iclealizada de los mercados incernacionales, suponiendo que son estables y que cs fácil

* Artículo publicado en la Rerista Mensaje, No 253, octubre de 1976, Santiago de Chile. Estudios Internationales lo reproduce con la attorización del autor. 
entrar a ellos. Para esta concepción, que constituye un extremo dentro de la gama de posibilidades en el terreno de politica económica, los procesos de integración no tienen razón de ser.

Los esquemas de integración tienen razón de ser, precisamente, para proveer un mercado ampliado a la sustitución de importaciones y para obtener un acceso más estable $y$ expedito para las exportaciones nacionales en los mercados de los países socios "; simultáneamente, el hecho de contar con un mercado más amplio y estable, facilita la salida posterior a mercados de terceros países. Finalmente, los acuerdos de integración sirven para acrecentar la capacidad de negociación de las naciones participantes en las muchas instancias en que a ellas les toca intervenir en la economía internacional.

El acuerdo de integración de los países andinos ha surgido de las expleriencias arrojadas por ALALC y de la conciencia entre los primeros de que un proceso intenso de integración permite remover obstáculos que enfrenta un desarrollo sostenido y acelerado, dentro de un marco de afirmación de la soberanía. El Pacto Andino es compatible con la permanencia de los seis países andinos en ALALC. Estos procuran avanzar más rápidamente para constituir un mercado común de la "subregión andina". Más adelante, sin perjuicio de las relaciones bilaterales que se puedan establecer en aspectos especificos, cuando los restantes miembros de ALALC estén dispuestos a intensificar este proceso de integración, los países andinos participarían en él como una sola unidad económica.

Las naciones andinas tienen. en, conjunto una gravitación comparable con la de los países grandes de ALALC. Todos los miembros del Acuerdo de Cartagena son países pequeños; no obstante, como se puede apreciar en el cuadro ačjunto, sumados alcanzan una magnitud respetable dentro del marco latinoamericano. En efecto, su dimensión es cercana a la del Brasil o a la de México. Por otra parte, el producto per cápita andino supera al del Brasil. Por último, la comparación es ampliamente más favorable para los países andinos cuando se refiere al volumen de las exportaciones: aún antes del alza del precio del petróleo, alcanzaban un monto similar al de las ventas en el exterior de la Argentina, el Brasil y México en conjunto. En síntesis, siempre que actúen unidos, los miembros del Pacto Andino disponen de la base de sustentación económica suficiente para tratar de igual a igual con cualquiera de los grandes paises latinoamericanos.

En el hecho, los mercados externos no son de fácil acceso. Ellos

1Un análisis más extenso se encuentra en un trabajo del autor, El Pacto Andino: un modelo original de Integración, Estudios de Planificación, No 42, Santiago, diciembre de 1974 . 
son. Ios mercados internos de los demás paises del mundo. Hay inestabilidad en muchos de ellos; todos los países aplican derecho de aduana y diferentes restricciones a sus importaciones; es un dato incontrovertible que parte de los mercados son cautivos de las corporaciones transnacionales. Por consiguiente, es difícil exportar una serie de productos no tradicionales en forma estable y conveniente para el país. La integración económica da acceso a mercados más amplios que el nacional, io que permite aprovechar economías de escala en los rubros en los cuales los mercados del resto del mundo son difíciles. Para el empresario nacional, en cualquier país en desarrollo, es muy importante contar con información y certidumbre respecto de dónde vender; ello reviste particular importancia con un mercado interno tan deprimido como el chileno en la actualidad y difícil de predecir cómo va a evolucionar.

\section{EL ARANCEL EXTERNO COMÚN}

El Arancel Externo Común es el instrumento que defiende a los pro* ductores regionales frente a la competencia del exterior. Tanto el nivel que él alcance como la estructura que tenga puedien influir en forma apreciable sobre el desarrollo de las economias andinas. ${ }^{2}$

Antes de entrar de lleno al tema, corresponde insistir en lo peligroso que resulta el hábito de un grupo de colegas economistas de plantear alternativas extremas como las únicas posibles en la política económica. Por un lado, presentan una alternativa idlealizada a la que le asignan todo tipo de virtudes; luego sostienen que cualquier otro camino congrega toda suerte de crrores e ineficiencias. Así por ejemplo, aparecen presentando como defensores del proteccionismo más burdo a los que critican las metas arancelarias propugnadas por el equipo económico. Es obvio, sin embargo, que hay una gran cantidad de economistas, en Chile y fuera de él, que rechazan por igual

2El arancel externo común, en virtud de lo acordado por los scis palses en marzo, debe aprobarse durante 1977 y empezar a regir en diciembre de 1978. Para el lapso intermedio que se extiende hasta 1978, se ha examinado por los cinco paises, en su reunión de Sochlagota (Colombia), un arancel mínimo que se aplique en este bienio. Este arancel comprende tasas descle 0 hasta 60 por ciento. Estos niveles reflejan la buena disposición de aquellos paises que estaban postulando tasas más elevadas, y un nivel hacia el cual han convergido los cinco paises, a pesar de sus diferentes regímenes políticos $y$ económicos. Refleja ello la conciencia que tienen todos de la importancia que para su desarrollo soberano reviste el proceso de integración en países pequeños como los nuestros. 
un librecambismo $y^{\prime}$ un proteccionismo excesivo, y que además sostienen la conveniencia de clectuar ajustes aruncelarios realmente gracluales, que eviten cierres o liquiclaciones de empresas y clesempleos costosos no sólo para los trabajadores afectados sino también para la economia chilena.

\section{LAS ECONOMIAS ANDINAS EN 1972}

(wulores cn moneda de 197.1)

\begin{tabular}{|c|c|c|c|c|c|}
\hline \multirow[b]{2}{*}{ Paíscs } & \multirow[b]{2}{*}{$\begin{array}{l}\text { Población } \\
\text { (millones) }\end{array}$} & \multicolumn{2}{|c|}{ I'roducto geográfico bruto } & \multicolumn{2}{|c|}{ Comercio exterior } \\
\hline & & $\begin{array}{c}\text { per capita } \\
\text { (dolares) }\end{array}$ & Total & $\begin{array}{l}\text { Exportaciones } \\
\text { (millones } \\
\text { ale dólares) }\end{array}$ & $\begin{array}{c}\text { Importa } \\
\text { ciontes }\end{array}$ \\
\hline & (1) & (2) & (3) & (4) & (5) \\
\hline Bolivia & 4.9 & 397 & 1.046 & 288 & 278 \\
\hline Colombia & 23.8 & 735 & $17.46 t$ & 1.3929 & 1.157 \\
\hline Chile & 10.1 & 1.248 & 12.565 & 1.214 & 1.710 \\
\hline Ecuador & 0.4 & 675 & 4.346 & 459 & $52 \mathrm{I}$ \\
\hline Pert & 14.5 & $75 \cdot 1$ & 10.917 & 1.343 & 1.193 \\
\hline Venezucla & 11.5 & $3.60 \%$ & is.455 & 5.393 & 3.327 \\
\hline Subregrión Anclina & 71.2 & 923 & 65.693 & 10.020 & 8.186 \\
\hline Argentina & 25.1 & 2.027 & 50.883 & 2.756 & 2.853 \\
\hline Brasil & 98.7 & 800 & 75.904 & 5.596 & 5.954 \\
\hline Mréxico & 54.3 & 1.926 & 72.287 & $2.64 t$ & 4.004 \\
\hline
\end{tabular}

FUENTE: Naciones Unidins, Anuario Estadístico de América Lalina, 1973 y Estudio Econó-

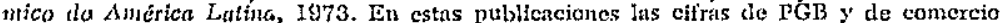
exterior están expresadas en dólaros de $19603^{*}$ de 1972 , respectivatnenle. Las primeras so hun multiplicado aquif por $1,90 \mathrm{y}$ ins segundas por 1,42 , para transformarlas en maneda de poder adquisitivo de 1974 . Los dos camisios principales acontecidus entre

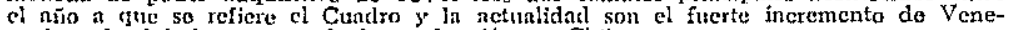
zucla $y$ la drístice memna do la producción en Chile.

El ministro de Economia ha afirmado que "El esquema andino significaría repetir en una escala mayor los mismos errores ya cometiclos en nuestro país" en el campo cle la sustitución de importaciones. Dentro de la misma tónica, otros declaran que "el nivel de protección arancelaria propuesta por los otros paises del Pacto Anclino conclena a nuestro país tal vez para siempre, a una sicuación de aislamiento e ineficiencia, descle el momento on que las actividades exportadoras no tradicionales no tendrian posibilidad alguna de desarrollarse". Una mera lectura de los documentos elaborados por la Junta del Acuerdo de Cartagena (JUNAC), preparaclos luego de frecuentes consultas a técnicos de todos los paises, demuestra que ella está plenamente consciente de que en cl pasado se han cometiclo errores en el proceso de inclustrialización de nuestros paises. Pero, con 
un estilo pragmático, en lugar de pretender deshacer lo avanzado durante estos decenios, busca remediar esos errores, discernicndo entre los aspectos posilivos y los negativos del proceso.

La propuesta andina de Arancel Externo Común elimina causas importantes de los clefectos pasalos: no contempla prohibiciones ni cuotas de importación, que lan sido dos factores determinantes de las ineficiencias registradas en el pasado. Los niveles arancelarios propuestos, de hasta $65 \%$, son decididamente moderados para el grado de desarrollo de nuestros países (los paises andinos han procurado que la teoría se adapte a cacla realidal y no viceversa). Tambićn lo son respecto de los vigentes en otros países latinoamericanos; por ejemplo, los aranceles medios aplicados en la actualidad por Brasil a los bienes de consumo son superiores a $50 \%$; de igual manera son moderados respecto de los que regian en cada país andino en $1975 .^{3}$

Los únicos aranceles relativamente altos corresponden a algunas producciones asignadits a Bolivia y a Ecuador $y$ a la armaduria de automóviles. Lo primero se ha hecho con la aprobación unánime de los paises, con el oljecto de compensar a Bolivia y Ecuador su calidacl de menor desarrollo económico. *

De estos antecedentes fluye que no tiene ninguna base la afirmación de que la aplicación del arancel andino provocaría electos catastróficos sobre la capacidad exportadora. La realidad es al revés, como las crecientes exportaciones no traclicionales hacia los mercados andinos lo estín demostrando. Es preciso observar no sólo su nivel absoluto hoy día, alrededor de 100 millones de clólares, sino también considerar que antes de la firma del Acuerdo de Cartagena no alcanzaban a 19 millones. Cabe advertir, que la distinción según tipo de exportación reviste gran importancia. Las tradicionales, como el cobre, son cle fácil colocación. En cambio, es difícil encontrar mercados para muchas exportaciones nuevas. Ahi resicle uno de los puntos en los que las ventajas del Pacto Anclino son difícilmente superables.

No sólo el nivel, sino que también la estructura del arancel (Ias diferencias entre las tasas que se apliquen a clistintos productos de importación) posee importancia. La propuesta andina de arancei

3Esto biltimo es particuiarmente cierto cuando se trata de la llanada protección efectiva. Lia propuesta de Arancel Extcrno Comủn general preparada por la JUNAC se lar elaborado con tasas de protección efectiva de hasta 85 por ciento. Por ello resulta inexacto e incorrecto sostencr que la propuesta contenga protecciones efectivas de 215 por ciento.

4Respecto de los aranceies ent discusión para el sector atutomotriz, personeros del equipo económico parecen considerar atı la protección efectiva andina propuesta para la armadura de autos; curiosamente, la que han establecido recientemente en Chile es muclso mayor. En camisio, en la proposición andina se otorga una protección mís alta que la vigente en Chile a la producción de partes y piezas, por considerarse csta actividad más valiosa que la mera armadurfa. 
externo común está graduada, entre otros, por la capacidad de absorción de empleo de cada actividad. Así, comprende niveles más elevados de protección para las actividades intensivas en el uso de trabajo, y bajas para las que usan mucho capital. Por ejemplo, contempla tarifas mayores para la metalmecánica $y^{r}$ menores para la petroquímica y fertilizantes. De esta manera, se procura que las nuevas inversiones absorban más empleo por unidad de capital. En un país con una desocupación tan dramática como la que Ghile sufre en estos años, al sector industrial le corresponde un rol destacado en la generación de empleos productivos; los países andinos ya proveen un mercado importante y mucho mayores son las perspectivas futuras. Por ejemplo, las posibilidades de colocación de exportaciones del sector metalmecánico son muy promisorias dentro del Pacto Andino, como muy bien lo ha señalado ASIMET. La insistencia del equipo económico en continuar su liberación de importaciones, en especial en las actuales condiciones de depresión de la industria nacional, resultará dañina no sólo para muchas industrias sino también para el nivel de ocupación. En efecto, es fácil provocar cierres de fábricas con una liberación excesiva; en cambio, es lenta y difícil la creación y expansión de las nuevas cmpresas.

Aun cuando las rebajas anunciadas para el futuro son muy fuertes $-y$ serán crecientemente más dolorosas en lo que sigue- ya se han dejado sentir los efectos de una liberación excesiva. Ello se aprecia en la profusión con que se ha empezado a hablar de dumping. El problema de la intensidad se ha agravado con la drástica reducción que ha experimentado el tipo de cambio real en lo corrido del año que, al incluir la revaluación del 29 de junio, alcanza a un deterioro superior a $25 \%$. ¿Son compatibles la liberación de importaciones al ritmo anunciaclo, con una reactivación de la economía y con el actual tipo de cambio?

INVERSIÓN EXTRANJERA Y DECISIÓN 24

La Decisión 24 no busca la nacionalización total, sino tan sólo la conversión en mixtas de las empresas extranjeras ( $51 \%$ de capitales nacionales, o $90 \%$ si es el Estado el socio) sin perjuicio de que el país que lo juzgue necesario puecla ir más allá. Se pretende así, que una mayor proporción de los beneficios potenciales se transmitan hacia los productores nacionales -accionistas, administradores, empleo, difusión de tecnologia- de forma que dejen un efecto positivo 
permanente en la economía nacional. La conservación por el inversionista extranjero de un $49 \%$ o un $70 \%$ de la propiedad, según el caso, mantiene su interés en la operación de la empresa. A su vez, la participación nacional directa debe posibilitar que los aportes de tecnología sean más valiosos que lo que fueron en el pasado. Véase, por ejemplo, la naturaleza limitada de los aportes efectuados por la inversión extranjera con la aplicación de un Estatuto generoso como era el DFL 258, de 1960. De algo deben servir las lecciones del pasado.

La conversión en mixta no requiere necesariamente retiros de capitales del país. Ese proceso puede realizarse mediante la participación nacional en las expansiones de inversiones. Las oportunidades de inversión en la economía chilena, con un mercado tan deprimido, no son muy claras actuaimente; una excepción es la que ofrece el mercado andino en muchos rubros. Indudablemente, las empresas que se conviertan en mixtas requerirán fuertes inversiones para producir para el mercado andino, tantas veces superior al nacional. Allí habría un nuevo destino productivo para el ahorro nacional que hoy no encuentra una ubicación estimulante.

La actitud de las corporaciones transnacionales frente a la asociación con capitales y gestión de los paises en desarrollo ha ido cvolucionando en forma positiva, presionada por tendencias registradas en muchos de estos paises. En Ia actualidad, hay mayores posibilidades objetivas para la constitución de empresas mixtas. En consecuencia, el desarrollo reciente de la economía internacional, en lugar de restarle vigencia, le da mayor validez a la Decisión 24.

Por otra parte, la conversión en mixta no es obligatoria en los sectores de productos básicos (mineros y forestales). Reflejando la flexibilidad que caracteriza en general a la Decisión 24, cada país puede decidir si recurre a empresas foráneas o no, en los sectores que por su propia naturaleza están poco ligadas al proceso de integración. Lo mismo sucede respecto de la conversión de la inversión que destine más de un $80 \%$ de su producción a mercados de terceros países.

Por último, la posibilidad de que el capital proveniente de cualquier pais andino llegue a ser computado como nacional, para cumplir con las normas de conversión en mixta, facilita el desarrollo de empresas subregionales. Debe destacarse, en este sentido, la oportunidad de crear empresas multinacionales andinas, que serán regidas por la Decisión 46, que acaba de entrar en aplicación luego de su reciente ratificación. La constitución de empresas multiandinas permitiria entrar a competir en un mejor pie en los mercados externos (proveyendo asi otra fuente de incremento de las exportaciones no tradicionales) y contribuir a un avance tecnológico propio. 
Esta recolccción muy escueta demuestra qua la Decisión 24 no es un marco inflexible. Se la ha atacado al bulto, sin adentrarse lo suficiente en sus disposiciones. La Decisión 24 no refleja fobia, pero tampoco idolatría ni idealización de los efectos del capital foríneo. Por el contrario, la norma es flexible y pragmática, y reconoce que hay buenas $y$ malas inversiones extranjeras. Por cllo se establece la necesidad de seleccionar entre ellas. Por lo mismo, es preciso ser estricto en el sentido de secoger para nucsuto país la ventaja que provee el Pacto Andino: el inversionista que entra a dil cuenta con seis mercados en lugar de uno, con una dimensión que representa alrededor cle 6 veces el de Chile (en la actualidad, debido a la deterioracla situación cconómica interna, cs entre 7 u 8 veces). Lat mayor dimensión del mercado pucde ofrecer mucho más atractivo, que una entrada indiscriminada del inversionista cxtranjero a un mercado pequeño y distante, hecho que pesa en las decisiones de cualquier inversionista. La aplicación cle reglas claras, estables y razonablemente exigentes parece funcionar aclecuadamente para atraer a la inversión extranjera que se caracterice por aportes más positivos. Ello es respaldado por el comportamiento de la inversión extranjera en Colombia, que ha llevado a grupos empresariales y gubernamentales de ese pais a sostener la vigencia de los aspectos esenciales de la Decisión 24. Por el contrario, normas demasiaclo favorables y una actitud de apertura irrestricta son de dudosa estabilidad. Por ello atraen a los inversionistas menos serios o que están dispuestos a correr riesgos: pero a ganar mucho a corto plazo. De ahí la gran presión que han ejercido algunos sectores para modificar el tope de $14 \%$ anual sobre las remesas de utilidades al exterior. Con cualquier patrón de comparación, una tasa anual cie miliclad líquicla real deI $14 \%$ resulta claramente razonable. Los países han aceptado elevarla a $20 \%$, que es una tasa muy altá. Sin embargo, lo importante es que el límite sobre las remesas actúa sobre el resultado final en Iugar de haccrlo sobre las causas de utilidades excesivas. Por ello resulta importante la aplicación efectiva de las restantes disposiciones de la Decisión 24, pues son las que permiten controlar las causas de utilidades que las elevan a montos superiores a un retorno normal de las inversiones extranjeras y conducen a acentuar los rasgos positivos que éstas posean. 
Se trata de un momento crucial del desarrollo del Pacto Andino. Nuchas acciones están en marcha, con inversiones realizándose en todos los países y' con asignaciones de producción decidiéndose en los diversos progranas sectoriales de desarrollo inclustrial (electrónica, automotriz, siderúrgico, vidrio, químico, etc.). Puesto que no cabe pensar que Chile se desarrolle en forma equilibrada al margen de los otros cinco hay que examinar las consecuencias cle una posición inflexible que conduzca a la ruptura. Se está en un momento de conformación de nuevas estructuras de producción dirigiclas al mercado subregional. Aun la vilelta futura en las condiciones más favorables posibles, clificilmente dejarian a Chile en pic tan conveniente como podría scrlo seguir hoy en la integración con los otros paises. Por lo tanto, una conccpción que descansa excesivamente en el libre comercio podría causarle grave claño a la nación, si lleva al extrcmo de la ruptura.

En el área de la inversión extunjera, de la politica arancelaria y de la programación inclustrial, es indudable que hay perfeccionamientos que aportar; asimismo hay otras decisiones que deben adop. tarse en el futuro, en las que puede irse modelando la evolución del proceso de integración andina. Para ser cficaz, se requiere un examen serio de cada paso, sin caer en extremismos económicos. Lstos sólo sirven para generar antagonismos entre paises y para quitarle sentido constructivo a la discusión.

Ojalí surja en nuestro país una posición menos tcorizante, que Ie permita continuar dentro del proceso $y^{r}$ contribuir positivamente a su desarrollo. Hay buenas y malas integraciones. La del Pacto Andino ha recogido mucho de las experiencias pasaclas de la región, lo que la hace un esquema que, en general, estai aclectado a las necesidades y características de un pais con el nivel de desarrollo y dimensiones de la economia chilena. Por ello sería lamentable para Chile su marginación, por temporal que sea. Lat integración andina no consticuye una panacca para todo; pero un proceso dinámico. como lo ha sido hasta hoy y como se perfila en los próximos años, puede contribuir en forma positiva a la recuperación de un ritmo más satisfactorio de crecimiento y a un desarrollo soberano mús sostenido. 